\title{
edmetic
}

Revista de Educación Mediática y TIC

Redes sociales aplicadas a la educación: EDMODO

Applied social networks for education: EDMODO

Fecha de recepción: 25/07/2014

Fecha de revisión: 13/11/2014

Fecha de aceptación: 21/03/2015 


\section{Redes sociales aplicadas a la educación: EDMODO}

Applied social network for education: EDMODO

\section{Santiago Alonso-García', Encarnación Morte-Toboso² \& Sebastián Almansa- Núñez ${ }^{3}$}

\section{Resumen:}

El artículo que a continuación abordamos, versa sobre un proyecto de innovación educativa que trata de incorporar las Redes Sociales a la educación analizando su valor didáctico. Esto se realiza a través del aprendizaje y posterior uso de una plataforma educativa llamada EDMODO.

Abordaremos el tema del aprendizaje mixto o blended learning, facilitando su concepto y las ventajas que esta metodología aporta al proceso de enseñanza-aprendizaje. Finalizaremos este primer apartado analizando de manera global el concepto de comunidades virtuales y las ventajas que éstas proporcionan a la práctica docente. De forma más detallada, nos centraremos en una de estas comunidades virtuales, llamada EDMODO, en la cual se centra nuestro proyecto de innovación. Una vez examinado el marco teórico de este proyecto, pasaremos a tratar los objetivos, la metodología y la aplicación del mismo. Llegados a este punto, tendremos la información necesaria para analizar los resultados positivos obtenidos y extraer las conclusiones oportunas sobre la necesidad de aplicación de las redes sociales en contextos educativos, concretamente EDMODO. Para terminar este proyecto, analizaremos las futuras líneas de implantación de estas redes sociales en los centros educativos y su futura viabilidad.

\footnotetext{
1 Universidad Nacional de Chimborazo (Ecuador). santiagoalonso@unach.edu.ec

2 Consejería de Educación de la Junta de Comunidades de Castilla la Mancha. encarmt@gmail.com

3 Consejería de Educación de la Junta de Comunidades de Castilla la Mancha. sebas351983@gmail.com
} 


\title{
Palabras claves:
}

TIC, educación a distancia, educación mixta, Web 2.0

\begin{abstract}
:
The article then boarded, concerns a project of educational innovation that seeks to incorporate social networks to education. This is done through learning and subsequent use of an educational platform called EDMODO. We address the issue of blended learning or blended learning, facilitating its concept and the advantages that this methodology provides the teaching-learning process. We end this first section comprehensively analyzing the concept of virtual communities and the benefits they provide to the teaching practice. In more detail, we will focus on one of these virtual communities called EDMODO, where our innovation project focuses. After examining the theoretical framework of this project, we will address the objectives, methodology and implementation. At this point, we will have the information necessary to analyze the results and drawing conclusions on the application of social media in educational contexts, specifically EDMODO. To complete this project, we will discuss future directions for the implementation of these social networks in schools and future viability.
\end{abstract}

\section{Keywords:}

TIC, E-learning, B-learning, Web 2.0. 


\section{Introducción}

1.1. Blended Learning (BL) O Aprendizaje Mixto

Frente a la enseñanza tradicional, en la era tecnológica surgida a finales del s. $X X$, surge el E-learning el cual da paso a una metodología mixta o lo que se conoce en el mundo anglosajón como blended learning, b-learning o BL.

En el año 2002 apareció el término "blended learning", que se traduce literalmente aprendizaje mixto, es decir, que pretende utilizar dos estrategias, la presencial y la virtual, por lo tanto, busca seleccionar lo mejor de cada una de ellas (Acosta, 2009).

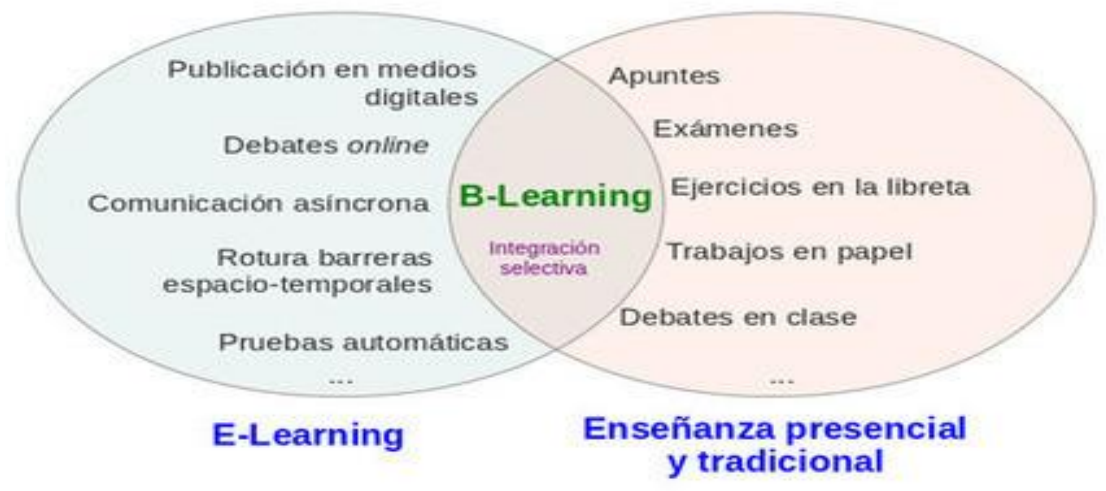

Figura 1: Del e-learning al B-learning

Bartolomé (2002), lo define como "un modelo que trata de recoger las ventajas del modelo virtual tratando de evitar sus inconvenientes. Aprovecha la importancia del grupo, el ritmo de aprendizaje y el contacto directo con el profesor de la enseñanza presencial, pero trata de desarrollar en los alumnos la capacidad de auto-organizarse, habilidades para la comunicación escrita, y estilos de aprendizaje autónomo. Especialmente importante en este modelo es el desarrollo de habilidades en la búsqueda y trabajo con información en las actuales fuentes de documentación en Internet".

Coaten (2003) y Marsh (2003), exponen el BL como el "modo de aprender que combina la enseñanza presencial con la tecnología no presencial". 
D. Randy Garrison y Heather Kanuka (2004), creen que radica en "La integración de las experiencias de aprendizaje cara a cara con las experiencias de aprendizaje on-line".

Charles R. Graham (2005), lo visualiza como "Un sistema Blended Learning es la combinación de instrucción desde dos modelos de enseñanza y aprendizaje, separados históricamente: los sistemas de aprendizaje tradicional cara a cara y los sistemas mediados por ordenador."

También señala que el blended-learning "es aquel diseño docente en el que tecnologías de uso presencial (físico) y no presencial (virtual) se combinan en orden a optimizar el proceso de aprendizaje" (Bartolomé, 2006).

Richard C. Larson y M. Elizabeth Murray (2008), conciben el BL "La integración planificada de enfoques de educación cara a cara y on-line de manera que se maximicen las características positivas de cada modo de entrega respectivo"

Morán (2012), lo define como la "modalidad de enseñanza que integra y articula los mejores elementos de ambos entornos (presencial/online) en los cuales se desarrolla la enseñanza y el aprendizaje para poner de manifiesto su potencial riqueza"

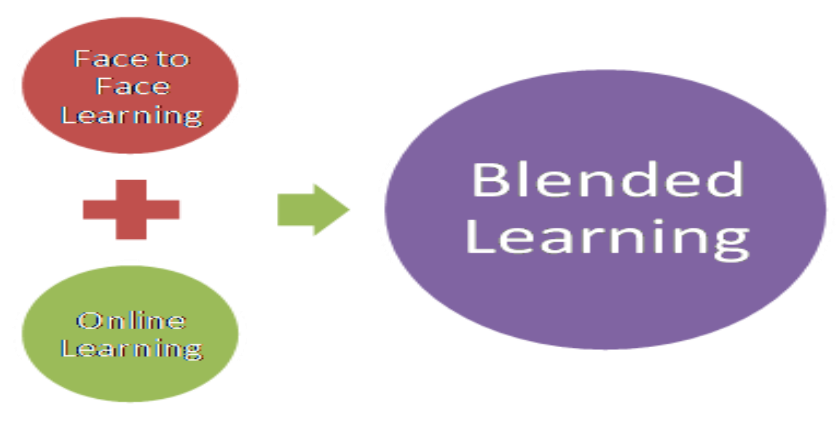

Figura 2: B-learnig

Así pues, el BL combina dos tipos de aprendizaje, presencial y virtual. En cada uno de éstos podemos diferenciar las siguientes dimensiones:

Configuración del espacio y del tiempo: aquí se integran los aspectos 
relativos al uso del tiempo y del espacio que se realiza en cada uno de los entornos.

Proceso de enseñanza y de aprendizaje: hace referencia a los aspectos específicos de la propuesta de enseñanza-aprendizaje que caracteriza a la formación en un entorno presencial y en un entorno online.

Socialización: hace referencia al contacto que establecen quienes participan de la formación (alumnos y docentes). Ambas formaciones (presencial y online) poseen unas características que podemos ver en la siguiente tabla: 
Tabla 1: f. presencial vs f. a distancia

Fuente: Elaboración propia

\begin{tabular}{|c|c|c|}
\hline DIMENSIONES & FORMACIÓN PRESENCIAL & FORMACIÓN ON LINE \\
\hline $\begin{array}{l}\text { CONFIGURACIÓN } \\
\text { ESPACIO Y DEL TIEMPO }\end{array}$ & $\begin{array}{l}\text { - Los tiempos y espacios se } \\
\text { corresponden con los del } \\
\text { aula. }\end{array}$ & $\begin{array}{l}\text { - En cualquier momento y } \\
\text { lugar. } \\
\text { - Permite la regulación de los } \\
\text { tiempos personales }\end{array}$ \\
\hline $\begin{array}{l}\text { PROCESO DE ENSEÑANZA Y } \\
\text { APRENDIZAJE }\end{array}$ & $\begin{array}{l}\text { - Los procesos de enseñanza } \\
\text { y aprendizaje se inician y se } \\
\text { desarrollan en el contexto de } \\
\text { las clases presenciales. } \\
\text { - Hay espontaneidad en la } \\
\text { participación de alumnos y } \\
\text { docentes. } \\
\text { - Requiere la respuesta } \\
\text { inmediata de los participantes } \\
\text { en el proceso formativo. } \\
\text { - Se utilizan materiales que se } \\
\text { caracterizan más por su } \\
\text { extensión que por su } \\
\text { diversidad. } \\
\text { - Se prioriza la oralidad sobre } \\
\text { la escritura }\end{array}$ & $\begin{array}{l}\text { - Mayor autonomía en el } \\
\text { proceso formativo. } \\
\text { - Se permiten ajustes en los } \\
\text { programas de formación } \\
\text { durante el curso. } \\
\text { - Se cuenta con un registro } \\
\text { de todas las intervenciones y } \\
\text { participaciones que se } \\
\text { pueden recuperar en } \\
\text { cualquier momento y lugar. } \\
\text { - Se dispone de un tiempo } \\
\text { mayor para elaborar las } \\
\text { participaciones, pensar y } \\
\text { comprender. } \\
\text { - Se distingue por la } \\
\text { diversidad en el uso de } \\
\text { actividades y materiales. } \\
\text { - Se desarrolla de forma } \\
\text { prioritaria la escritura sobre la } \\
\text { oralidad. }\end{array}$ \\
\hline SOCIALIZACIÓN & $\begin{array}{l}\text { - Permite crear un vínculo } \\
\text { estrecho de contacto cara a } \\
\text { cara entre alumnos y } \\
\text { docentes. }\end{array}$ & $\begin{array}{l}\text { - Se produce el contacto con } \\
\text { otros participantes a través de } \\
\text { diversos canales de } \\
\text { comunicación. }\end{array}$ \\
\hline
\end{tabular}




\subsection{La Web 2.0 en el B-Learning}

Web 2.0 es un concepto que se acuñó en 2003 y que se refiere al fenómeno social surgido a partir del desarrollo de diversas aplicaciones en Internet. El término establece una distinción entre la primera época de la Web (donde el usuario era básicamente un sujeto pasivo que recibía la información o la publicaba, sin que existieran demasiadas posibilidades para que se generase la interacción) y la revolución que supuso el auge de los blogs, las redes sociales y otras herramientas.

De la Torre. (2006), define Web 2.0 como "una forma de entender Internet que, con la ayuda de nuevas herramientas y tecnologías de corte informático, promueve que la organización y el flujo de información dependan del comportamiento de las personas que accedan a ella, permitiéndose no sólo un acceso mucho más fácil y centralizado a los contenidos, sino su propia participación tanto en la clasificación de los mismos como en su propia construcción, mediante herramientas cada vez más fáciles e intuitivas de usar."

Xavier Ribes (2007), estima que "podemos entender como Web 2.0 todas aquellas utilidades y servicios de Internet que se sustentan en una base de datos, la cual puede ser modificada por los usuarios del servicio, ya sea en su contenido (añadiendo, cambiando o borrando información o asociando metadatos a la información existente), bien en la forma de presentarlos o en contenido y forma simultáneamente".

Sabin (2009), afirma que "la Web 2.0 se conforma de espacios virtuales que son abiertos, auto-organizativos, adaptativos, ágiles, accesibles y fáciles de usar, que cuentan con servicios de soporte colaborativo que permiten a los usuarios compartir, opinar o crear nuevos contenidos."

Según Deans, C. (2009), básicamente, "la Web 2.0 es la segunda generación de servicios y comunidades basadas en Web que facilita la colaboración entre usuarios".

En este sentido, Dans, E. (2009), afirma que "la Web 2.0 es una 
tendencia con un funcionamiento cada vez más participativo y bidireccional, en la que los alumnos no se limitan a leer o visualizar contenidos, sino que incrementan su nivel de implicación produciendo sus propios contenidos y publicándolos mediante sencillas aplicaciones. Por tanto, cada vez son más los profesores y estudiantes que comienzan a relacionarse y compartir conocimiento a través de las tecnologías propias de la Web 2.0".

Alberto Ortiz De Zárate (2009), se refiere a la Web 2.0 como "un fenómeno social en relación con la creación y distribución de contenidos en Internet, caracterizado por la comunicación abierta, la descentralización de autoridad, la libertad de compartir y usar, dentro de un enfoque que trata a las relaciones humanas como conversaciones".

Por último, Alonso y Alonso (2014: 57), estiman que La Web 2.0 es un fermento en el proceso didáctico en el que se unen las competencias y demandas sociales, con la institución y la persona asincrónicamente.

\section{3.- Comunidades virtuales en la educación: EDMODO}

Las comunidades virtuales de aprendizaje son redes sociales cuyo objetivo principal está relacionado con el aprendizaje. Internet proporciona espacios compartidos que permiten intercambiar información de manera eficaz, facilitando así la realización de actividades entre alumnos separados geográficamente, optimizando así, (Alonso y Palomares, 2013:162) el trabajo realizado en aspectos como el espacio, la temporización, las metodologías o los ámbitos (burocrático-funcional, personal, escolar, académico, profesional y docente).

El éxito de las redes sociales educativas radica en el interés que estos espacios ya despiertan por sí solos en la sociedad actual. Algunas de las ventajas que las redes sociales pueden aportan a la práctica docente son las siguientes: 
- Aumento del sentimiento de comunidad educativa para alumnos y profesores debido al efecto de cercanía que producen las redes sociales.

- Permiten centralizar en un único sitio todas las actividades docentes, profesores y alumnos de un centro educativo.

- Favorecen la comunicación entre los alumnos, especialmente cuando se forman grupos de trabajo incrementando así las habilidades sociales.

- Acerca la figura del docente a los estudiantes. El profesor ya no resulta tan inaccesible y su localización puede llevarse a cabo fácilmente a través de la red en lugar de otros medios.

- El aprendizaje resulta más satisfactorio para los estudiantes, incrementándose su grado de motivación ante la posibilidad de ser orientados por otros alumnos o de convertirse en instructores de sus propios compañeros.

- Las herramientas que proporcionan las redes sociales permiten al profesor hacer un seguimiento exhaustivo de su clase, de modo que se convierte en testigo del proceso de trabajo y aprendizaje de sus alumnos.

- Disponen de una amplia variedad de recursos educativos abiertos para estudiantes en formato digital. De esta manera, evitamos el consumo de papel y contribuimos a la reducción de costos en los centros educativos.

- Contribuyen a la formación integral de la persona, ya que se ponen en juego una serie de competencias y habilidades relacionadas con la colaboración, el espíritu crítico y la expresión creativa.

Una vez que hemos visto la definición y ventajas que las redes sociales tienen en la educación, nos centraremos en una plataforma social educativa llamada "EDMODO" sobre la cual basaremos nuestro proyecto de innovación educativa.

EDMODO es una plataforma social educativa gratuita que fue creada 
en el año 2008 por Jeff O'Hara y Nic Borg. Su objetivo principal es permitir la comunicación entre profesores y alumnos en un entorno cerrado y privado. Se basa en un servicio de redes sociales creado para ser usado específicamente en educación. Este servicio proporciona al docente un espacio virtual en el que se pueden compartir mensajes, archivos y enlaces, un calendario de aula, así como proponer tareas y actividades y gestionarlas.

EDMODO tiene dos ventajas fundamentales con respecto a otras redes sociales que pueden ser utilizadas en el ámbito educativo:

La primera ventaja es que los alumnos no necesitan dar ningún dato personal, más allá de su nombre, para utilizar la red social. El profesor crea un grupo privado y genera un código que será utilizado por los alumnos para acceder a la plataforma la primera vez.

La segunda ventaja es el control paternal: las familias pueden disponer de un acceso especial a la red social, gracias al cual pueden acceder al calendario, notas, mensajes del alumno y enviar o recibir mensajes del profesor.

Las funciones son muy diversas, entre las cuales EDMODO nos permite:

- Crear grupos privados con acceso limitado a docentes, alumnos y padres.

- Disponer de un espacio de comunicación entre los diferentes roles mediante mensajes y alertas.

- Compartir recursos multimedia tales como archivos, enlaces, vídeos, etc.

- Incorporar los contenidos de nuestros blogs.

- Hacer encuestas a los alumnos.

- Asignar tareas a los alumnos y gestionar las calificaciones de las mismas.

- Gestionar un calendario en clase.

- Crear comunidades donde agrupar a todos los docentes y alumnos de nuestro centro educativo. 
- Dar acceso a los padres a los grupos en los que estén asignados sus hijos y tener la posibilidad de comunicación con los profesores.

- Conceder insignias a los alumnos como premios a su participación en el grupo.

- Gestionar los archivos y recursos compartidos a través de la biblioteca.

- Crear subgrupos para facilitar la gestión de grupos de trabajo.

- Disponer de un espacio público donde mostrar aquella actividad del grupo que el profesor estime oportuna.

- Algunas de las características de esta plataforma educativa son:

- Acercamiento profesor/alumno.

- Incremento de la comunicación.

- Aumento del sentimiento de comunidad educativa.

- Facilita el aprendizaje (espacio común).

- Facilita la coordinación del trabajo.

- Se incrementa el aprendizaje informal.

- Fomenta y democratiza las relaciones.

- Enseñar a convivir a los alumnos en Internet, respetando su privacidad y la de los demás.

- Enseñar a respetar el trabajo y las ideas de los demás.

- Fomentar el trabajo en equipo, en colaboración con otros, mediante las redes sociales.

- Fomentar el autoaprendizaje, enseñando a establecer las conexiones necesarias entre compañeros.

Así pues, teniendo en cuenta el contexto y la justificación teórica, procederemos a seguir exponiendo los siguientes puntos de esta investigación.

\section{2.- Problema de investigación}

Centraremos esta investigación en el conocimiento de la incidencia de las redes sociales, en concreto EDMODO, sobre la calidad de los procesos didácticos, en base a la aplicación de un proyecto de innovación sobre dicha 
red social.

2.1. Objetivos

- Dar a conocer el blended learning o aprendizaje mixto.

- Incentivar el uso de la E-Tutoría.

- Impulsar el enfoque educativo de las redes sociales.

- Fomentar el uso didáctico de las TIC mediante la plataforma EDMODO.

- Promover futuras líneas de innovación e investigación.

\section{Metodología}

En ese sentido, y dado que la finalidad del presente proyecto radica en iniciar en el uso de EDMODO, resulta obvio que el cuestionario se muestra como una técnica de recogida de datos imprescindible, cuyo análisis cuantitativo refleja con gran precisión los recursos tecnológicos utilizados para el trabajo docente, la aplicación que se hace de los mismos, etc.

No obstante, aunque bien es cierto que la encuesta, en efecto, posibilita la obtención de una gran cantidad de resultados objetivos extraídos del análisis estadístico de los datos (cifras, porcentajes, etc.), resulta, sin embargo, un instrumento limitado cuando se quiere entrar en materia de comprensión y establecimiento de relaciones causales. A este proyecto de innovación, también le interesan las razones por las cuales las cosas son así, desde esa perspectiva, parece evidente que la técnica más adecuada para alcanzar tal fin es el grupo de discusión, cuya función principal consiste en complementar e ilustrar los resultados obtenidos cuantitativamente a través del cuestionario, esgrimiendo algunos de los posibles motivos de ocurrencia de la fenomenología estudiada.

Desde este punto de vista, abordamos para esta innovación/investigación una metodología de naturaleza empírica y cuantitativa, para lo cual, y tal y como señalábamos anteriormente hacemos uso de la encuesta estandarizada de recogida de datos para cuyo análisis y tratamiento haremos uso de la ciencia estadística en conjunción con el grupo 
de discusión que nos dará el punto de vista cualitativo necesario para dirimir aspectos poco apreciables en el análisis cuantitativo.

\subsection{Población - muestra}

El muestreo es, sin la menor duda, una de las etapas más importantes en el proceso de investigación científica. En este sentido y, como paso previo, cabe delimitar convenientemente unos cuantos conceptos esenciales para la formalización de nuestro estudio, a saber (Arnal. Del Rincón., Latorre, y Sans, 2003).

En nuestro proyecto el muestreo es no probabilístico discrecional, y población y muestra coinciden, es decir, que la investigación se han realizado sobre los 13 miembros de uno de los grupo-clase de $6^{\circ}$ de primaria del centro La Castilla de la JCCM.

\subsection{Análisis de datos}

La mayoría de los alumnos encuestados, un 92,3\%, considera que, gracias a redes sociales como EDMODO, las asignaturas les parecen más interesantes. Por el contrario, hay una minoría, un $7,7 \%$, que considera que este tipo de redes sociales no incrementa su interés por las asignaturas. Más detalladamente, podemos decir que las asignaturas son mucho más interesantes para el $69,3 \%$ de los alumnos, y bastante más para el $23 \%$. 


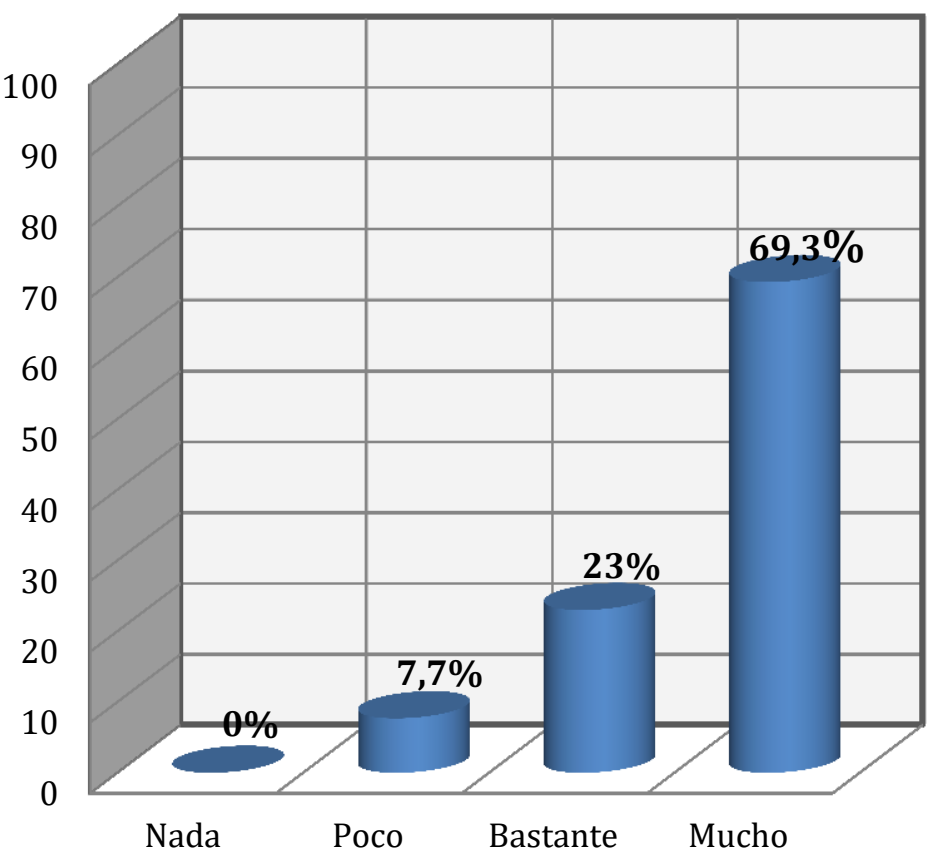

Gráfica 1: Las redes sociales como EDMODO hacen más interesantes las asignaturasmaterias

Fuente. Elaboración Propia

Un 92,3\% de los alumnos ha conocido EDMODO gracias a este proyecto (ver gráfica 2). Mientras tanto, hay un $7,7 \%$ de alumnos que piensa que este proyecto no les ha ayudado a conocer esta plataforma. Siendo más concretos, de ese $92,3 \%$, hay un $84,6 \%$ que lo han conocido muy bien, frente a un $7,7 \%$ que lo ha conocido bastante bien. 


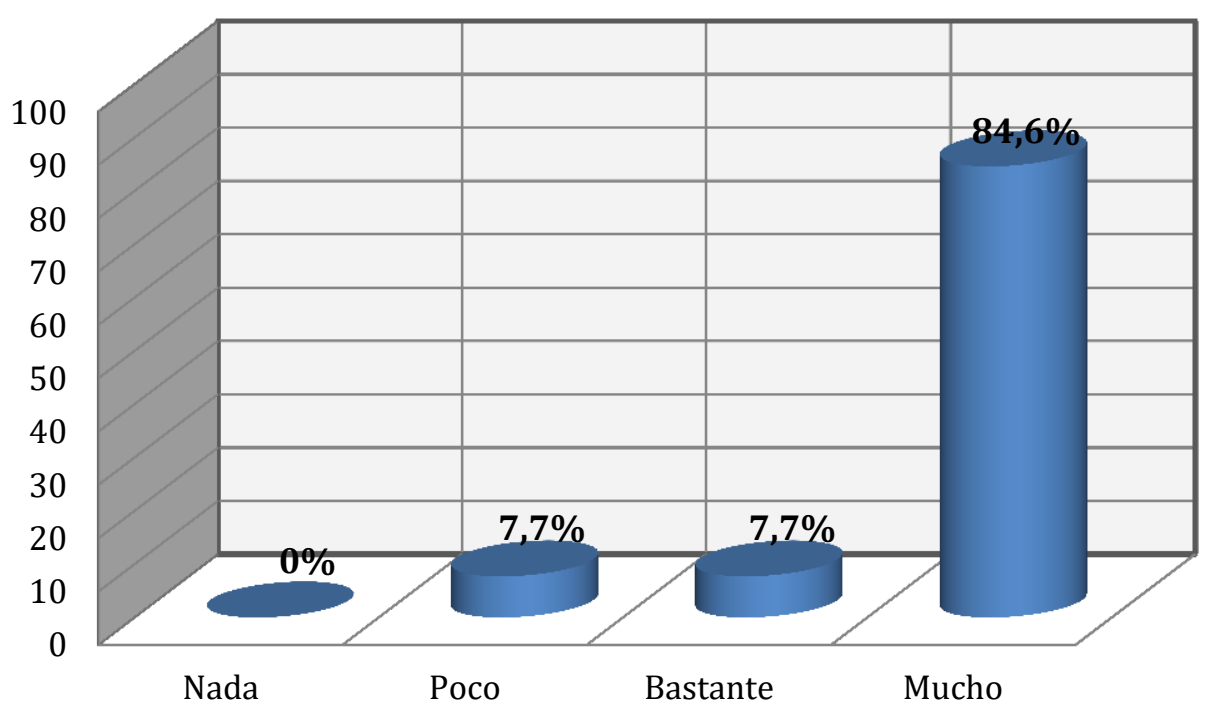

Gráfica 2: le proyecto me ha ayudado a conocer EDMODO Fuente: Elaboración propia

La gráfica 3 muestra que el 100\% de los alumnos ha aprendido a utilizar EDMODO a través de este proyecto. El 69,3\% de los alumnos opina que este proyecto les ha ayudado mucho a utilizar EDMODO, mientras que el 30,7\% opina que les ha ayudado bastante.

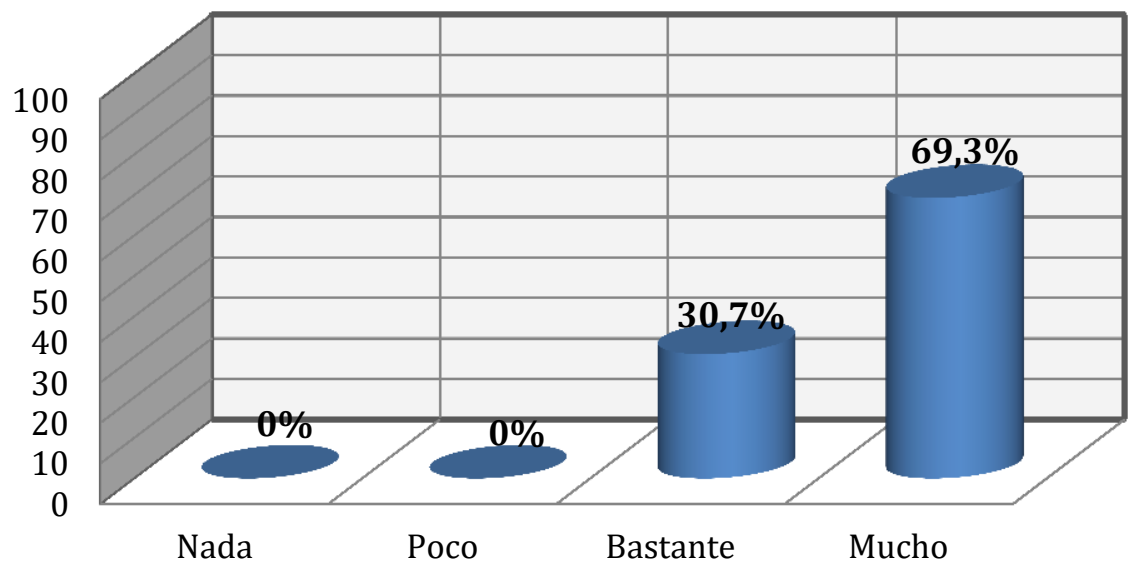

Gráfica 3: El proyecto me ha ayudado a utilizar EDMODO Fuente. Elaboración propia 


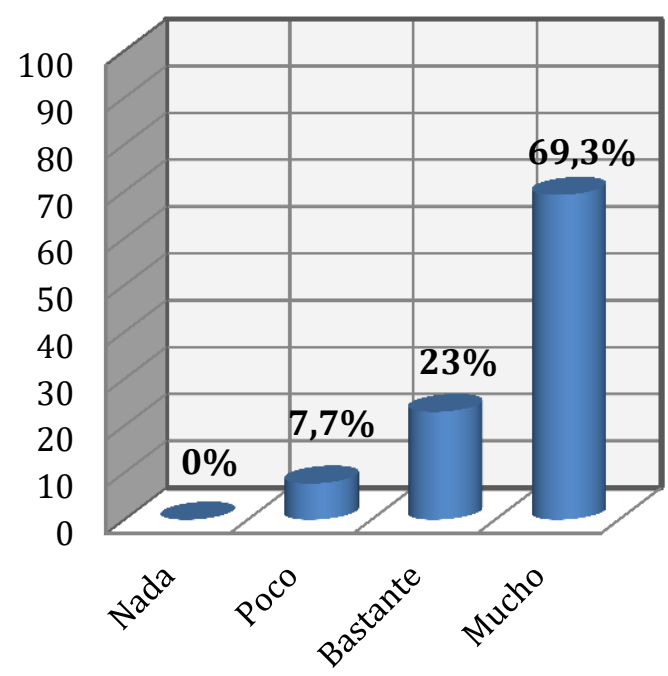

Gráfica4: La tutoría virtual complementa positivamente la presencial Fuente: Elaboración propia

Casi la totalidad de los alumnos, un $92,3 \%$, estima que las tutorías virtual y presencial se complementan positivamente. Por otro lado, hay un $7,7 \%$ que entiende que éstas se complementan poco. De los encuestados que respondieron positivamente a esta cuestión, hay un $69,3 \%$ que opina que las tutorías se complementan muy positivamente y un $23 \%$ que piensa que ambas tutorías se complementan bastante bien. 


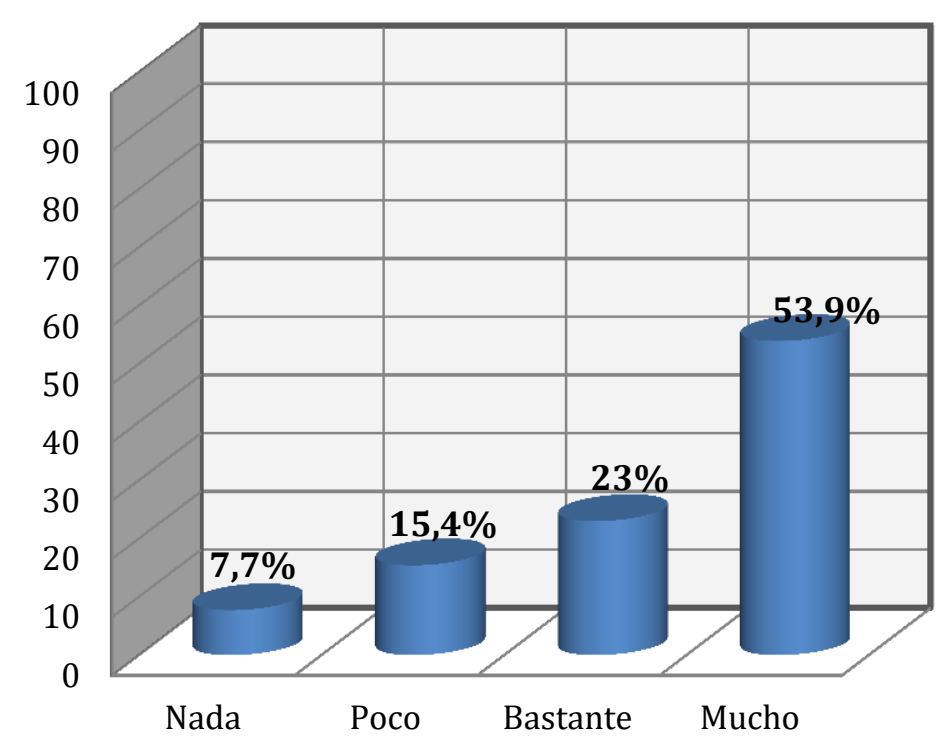

Gráfica 5: El uso de EDMODO hace que me interese más por las materias asignaturas

Fuente: elaboración propia

A través del uso de EDMODO, hay un $76,9 \%$ de alumnos que se interesan más por las asignaturas (ver gráfica 5). Sin embargo, un 23,1\% no encuentra más interesante las asignaturas usando esta plataforma. Los porcentajes mencionados anteriormente, podemos desglosarlos del siguiente modo: el $53,9 \%$ de los alumnos se interesan mucho más por las asignaturas usando EDMODO, el $23 \%$ se interesa bastante más, el $15,4 \%$ opina que el uso de EDMODO incrementa un poco su interés por las asignaturas.

En esta gráfica observamos que el $100 \%$ de los alumnos cree que EDMODO debe usarse en todas las áreas. El 69,3\% afirma que sería muy beneficioso, mientras que el $30,7 \%$ afirma que esto sería bastante beneficioso (ver gráfica 6). 


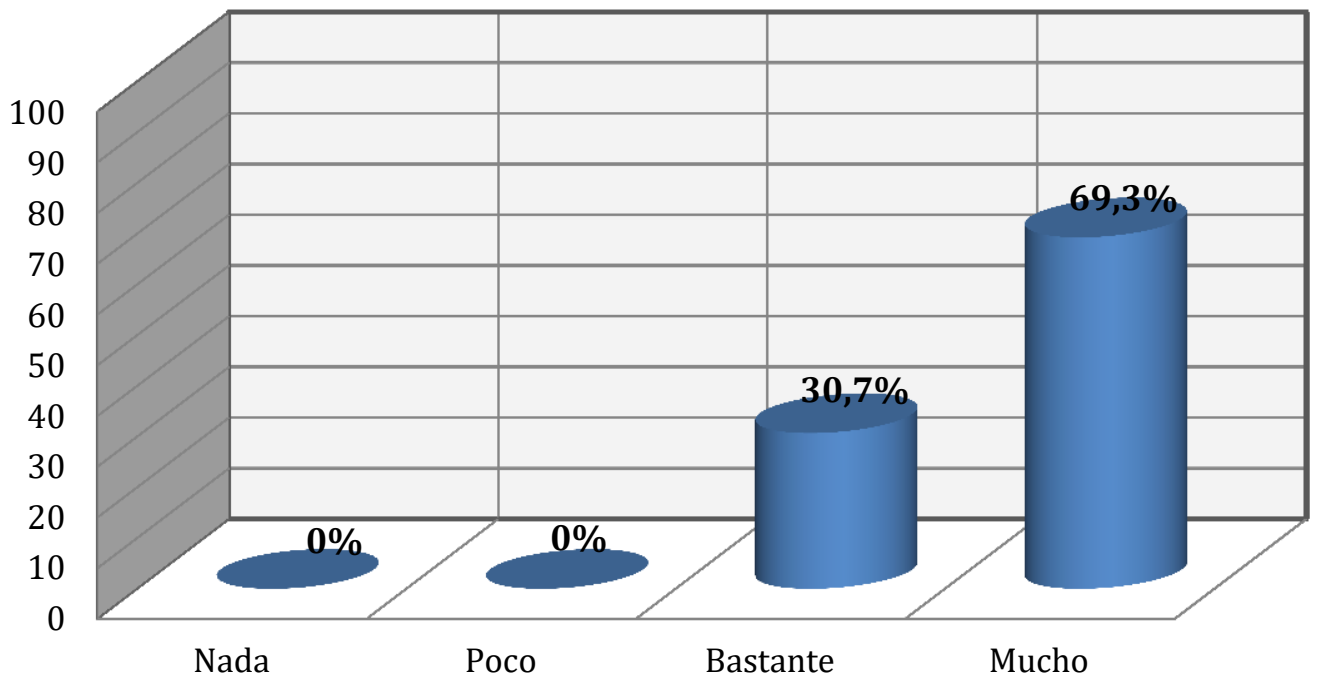

Gráfica 6. Creo que EDMODO debe usarse en todas las áreas-asignaturas Fuente: Elaboración propia

\section{4.-Conclusiones y resultados}

Primero (hemos mostrado el aprendizaje mixto con el uso del EDMODO) está relacionada con el objetivo que hace referencia a que los alumnos conocieran lo que es el blended learning o aprendizaje mixto. En nuestra opinión este objetivo ha sido conseguido en su totalidad, ya que durante el desarrollo de este proyecto hemos combinado sesiones y actividades que se realizaban en clase con otras que se realizaban desde casa. De esta manera, hemos conseguido combinar los dos aprendizajes en los que se basa el blended learning: presencial y virtual.

En el ítem 1 podemos observar que el 100\% de los alumnos estima que este proyecto, en el que se incluye dar a conocer a los alumnos lo que es el aprendizaje mixto, ha sido explicado con claridad por parte del profesorado. Esto también se puede comprobar con los resultados obtenidos en la gráfica 7 , en la que un $92,3 \%$ de los alumnos considera que la tutoría virtual complementa de manera muy positiva a la tutoría presencial.

La formación presencial se ha desarrollado durante las cuatro semanas 
que ha durado este proyecto. La primera semana la enfocamos a conocer de manera general lo que es el aprendizaje mixto y la plataforma EDMODO. La segunda y tercera semana las basamos en la realización de diferentes actividades para que los alumnos se familiarizaran con esta plataforma y fueran conociendo las distintas posibilidades que ésta nos ofrece. Para la realización de estas actividades en clase los alumnos han estado divididos en grupos, fomentando así el trabajo cooperativo y favoreciendo la socialización de los alumnos. Cada uno de ellos disponía de un ordenador portátil para la búsqueda de la información necesaria. La última semana del proyecto ha estado dedicada a la evaluación de éste, a través de encuestas y de un grupo de discusión que previamente habíamos preparado.

La valoración por parte del alumnado de la tutoría presencial ha sido muy gratificante como indican los resultados del ítem 5, ya que el 100\% de los alumnos opina que durante estas tutorías todas sus dudas han sido resueltas.

La formación virtual (on line) también se ha desarrollado a lo largo de las cuatro semanas de duración del proyecto. Esta formación ha sido una prolongación de la presencial. Los alumnos han terminado de realizar las actividades, individuales o grupales, que comenzaron en el aula a través de internet. Una vez finalizadas, los alumnos nos las mandaban a través de la plataforma EDMODO. Durante estas cuatro semanas de proyecto, nuestro rol ha sido el de guía, colaborador y facilitador del aprendizaje de nuestros alumnos.

\section{Segundo (hemos incentivado la E-tutoría mediante su practica con EDMODO)}

está directamente relacionada con el objetivo de incentivar el uso de la ETutoría. Single y Muller (1999), definen la e-tutoría como "la relación establecida entre un tutor y un alumno utilizando la comunicación virtual, con el objetivo de facilitar el desarrollo de habilidades y conocimientos, confianza y socialización del segundo, incrementando sus posibilidades de éxito".

Para conseguir este objetivo, durante la primera semana del proyecto fijamos con nuestros alumnos un horario en el que todos (alumnos y profesores) íbamos 
a estar conectados. El acuerdo al que llegamos entre todos fue dedicar dos horas semanales (martes y jueves) a la resolución de dudas o problemas (a través de EDMODO) que pudieran ir surgiendo, relacionados con la tarea a realizar o con el funcionamiento de la plataforma.

Consideramos que estas tutorías han tenido una gran importancia ya que los alumnos no sólo se apoyaban en sus profesores para resolver sus dudas, sino que todos los alumnos colaboraban en la resolución de las dudas y problemas que pudieran surgir a sus compañeros. Esta información la hemos podido corroborar con los resultados del grupo de discusión que se realizó en clase, ya que muchos de éstos han destacado el compañerismo existente al ayudarse los unos a los otros.

Como hemos podido comprobar al analizar los resultados de las encuestas (item 6) y el grupo de discusión, casi un 93\% de los alumnos han valorado de manera muy positiva el hecho de tener acceso a tutorías virtuales y poder resolver sus dudas desde casa.

\section{Tercero (hemos impulsado el enfoque educativo mediante su uso en clase)}

que sacamos con el desarrollo de este proyecto se refiere al enfoque educativo de las redes sociales.

Desde el primer día que empezamos a desarrollar este proyecto, tratamos de hacer ver a los alumnos que existen otro tipo de redes sociales distintas a las que, hoy en día, ellos conocen. En clase, argumentamos que no todas las redes sociales estaban únicamente destinadas a hablar con los amigos o a ver fotos de éstos, sino que había otro tipo de redes sociales destinadas al aprendizaje de los alumnos. Les explicamos que este proyecto estaba basado en una de estas redes sociales llamada EDMODO, que era una plataforma que íbamos a utilizar con fines educativos.

El simple hecho de trabajar con una red social provocó un gran interés y curiosidad en nuestros alumnos desde el primer momento. También la motivación de los alumnos aumentó al ser algo diferente a sus rutinas diarias.

A través del grupo de discusión se han confirmado las primeras 
impresiones que los alumnos tenían cuando comenzaron el proyecto. Muchos de ellos han contestado que el hecho de utilizar una red social a la hora de aprender les resultaba muy motivador.

El item número 2 confirma lo que hemos dicho anteriormente, ya que la gran mayoría de los alumnos encuestados, un 92,3\%, considera que el hecho de usar redes sociales educativas, hace que incremente su interés por las distintas áreas o asignaturas. Aun así, también hay un pequeño porcentaje, un $7,7 \%$, que opina que su interés por las asignaturas no ha aumentado incluso utilizando estas redes sociales.

Cuarto (EDMODO fomenta positivamente el uso de las TIC) hace referencia a fomentar el uso didáctico de las TIC mediante la plataforma EDMODO. Nuestra idea a la hora de plantear este objetivo era fomentar en los alumnos la idea de que las TIC no sólo tienen un uso lúdico, sino que también podemos usarlas con un fin didáctico y educativo.

Gracias al uso de la plataforma EDMODO, los alumnos han podido comprobar que también pueden aprender haciendo uso de las nuevas tecnologías.

Este proyecto ha brindado la posibilidad a los alumnos de ver que hay diferentes maneras de aprender, y que éstas pueden ser muy motivadoras e interesantes para ellos. El proyecto les ha ofrecido la oportunidad de comprobar que no sólo se aprende a través de los libros de texto y de las explicaciones del profesor en clase. Han aprendido que a través de las TIC, y haciendo un uso responsable y adecuado de éstas, pueden adquirir nuevos conocimientos o ampliarlos, e interesarse más por las diferentes asignaturas.

Esto puede comprobarse en el ítem 8, en la que podemos ver que una gran parte de los alumnos (cerca de un $80 \%$ ) piensa que gracias a EDMODO y al uso de las TIC su interés por las diferentes asignaturas ha aumentado. Esta información también puede ser corroborada en los ítems 3 y 4 , en las que un elevado porcentaje del alumnado (un $92,3 \%$ y un $100 \%$ respectivamente) opina que este proyecto les ha posibilitado conocer y utilizar EDMODO, y en la 
gráfica 9, en la que un $100 \%$ de los alumnos usaría esta plataforma en el resto de las asignaturas.

\section{Referencias bibliográficas}

ALONSO, S., Y ALONSO, M.M. (2014). Las redes sociales en las universidades españolas. Revista de Comunicación Vivat Academia, 126, 54 - 62.

ALONSO, S., Y PALOMARES, A. (2013). Percepciones de la función tutorial en el Espacio Europeo de Educación Superior del alumnado de la Facultad de Educación de Albacete (UCLM - España). Journal for Educators, Teachers and Trainers, 4(2), 160-168.

ARNAL AGUSTíN, J., DEL RINCÓN IGEA, D., LATORRE BELTRÁN, A., Y SANS MARTIN, A. (1995). Técnicas de Investigación en Ciencias Sociales. Barcelona: Dykison.

BARTOLOMÉ A., Y AIELLO M. (2006).Nuevas tecnologías y necesidades formativas Blended Learning y nuevos perfiles en comunicación audiovisual. España: TELOS.

BARTOLOMÉ, A. (2002). Universidades en la Red. ¿̇universidad presencial o virtuale Crítica, LII 896, 34-38.

COATEN, N. (2003). Blended e-learning. EducaWeb, 69. Monográfico sobre Formación Virtual.

DANS, E. (2009). Educación on-line. Plataformas educativas y el dilema de la apertura. Revista de Universidad y Sociedad del Conocimiento, 6(1), Recuperado el 05 de Febrero de 2010.

DE LA TORRE, A. (2006). Definición de Web 2.0. Bitácora de Aníbal de la Torre. 12 de abril 2006.

DEANS, C. (2009). Social software and Web 2.0 technology trends. USA: IGIGlobal.

GARRISON, D.R., Y Kanuka, H. (2004). Blended learning: Uncovering its transformative potential in higher education. The Internet and Higher Education 7(2), 95-105. 
GRAHAM, C.R. (2005). Blended Learning Systems: Definition, Current Trends, and Future Directions, en Handbook of Blended Learning: Global Perspectives, local designs, Pfeiffer Publishing, 3-21.

LARSON, R., Y MURRAY, M. (2008). Open educational resources for blended learning in high schools: Overcoming impediments in developing countries. Journal of Asynchronous Learning Networks 12(1), Sloan Consortium, 1-19.

MORÁN, L. (2012). Blended Learning. Desafío y oportunidad para la educación actual. EDUTEC. Revista Electrónica de Tecnología Educativa, 39.

RIBES, X. (2007). La Web 2.0. El valor de los metadatos y de la inteligencia colectiva. TELOS, Cuadernos de Comunicación e Innovación:

SABIN, M., Y LEONE, J. (2009). IT Education 2.0. In: Proceedings of the 10th ACM conference on SIG-information technology education, 2009. Virginia, USA

SINGLE P. B., Y MULLER C. B. (1999). Electronic Mentoring: Issues to Advance Research and Practice. Paper presented at the International Mentoring Association Conference, Atlanta, GA.

\section{Como citar este artículo:}

Alonso García, S., Morte Toboso, E., \& Almansa Nuñez, S. (2015). Redes sociales aplicadas a la educación: EDMODO. EDMETIC; Revista de Educación Mediática y TIC, 4(2), 88-111. 